\title{
OPTIMIZATION OF ELECTRO-CHEMICAL DISCHARGE MACHINING PROCESS USING GENETIC ALGORITHM
}

\author{
Ruben Phipon ${ }^{1}$ and B.B.Pradhan ${ }^{2}$ \\ Mechanical Engineering Department, SMIT, Sikkim, India.
}

\begin{abstract}
Machining process efficiency can be improved by optimizing the control parameters. This requires identifying and determining the value of critical process control parameters that lead to desired responses ensuring a lower cost of manufacturing. Traditional optimization methods are generally slow in convergence and require much computing time. Also, they may risk being trapped at local optima. Compared to these, Genetic Algorithm (GA) is robust, global and can be applied without recourse to domain-specific heuristics. Considering these novel traits of GA, optimization of Electro-Chemical Discharge Machining (ECDM) process has been carried out in this research. Mathematical models using Response Surface Methodology (RSM) is used to correlate the responses and the control parameters. The desired responses are minimum radial overcut (ROC) and minimum heat affected zone (HAZ) while the control parameters are applied voltage, electrolyte concentration and interelectrode gap. The responses are optimized individually as well as a simultaneous multiobjective optimization is solved. A Pareto optimal solution is the output result for multi objective optimization. Here each solution is non dominated among the group of predicted solution points thus allowing flexibility in operating the machine while maintaining the standard quality.
\end{abstract}

KeyWords - Electro-chemical Discharge Machining (ECDM), Genetic Algorithm (GA), Optimization, Pareto optimal, Response Surface Methodology (RSM).

\section{INTRODUCTION}

Electro-chemical discharge machining (ECDM) is a non-traditional machining process which combines the attributes of both electro-chemical machining (ECM) and Electro-discharge machining (EDM) [1]. There is no contact between the tool and the work piece in this machining process hence it does not require expensive alloys to make the tool tough like in the case of conventional machining. Also, the process requires fewer passes and the tool can be utilized repeatedly. This process can be used to machine hard and high strength to weight ratio materials. Also, intricate designs can be machined. The micro-electro-mechanical-system (MEMS) came into existence with the development of integrated circuits fabrication processes. Other technologies available for machining the required materials have difficulty to obtain good surface finish and are prone to potential micro structural damages. A possible solution for such machining is Electro chemical discharge machining [2].

To maintain the desired quality of machining products, to reduce the machining cost and to improve the machining effectiveness, it is very important to select the optimal machining parameters when the machine tools are selected. Thus, to meet the desired quality of responses, the process control parameters require to be set at their optimum values. This requires first to establish a mathematical model correlating the response and the control parameters. Thereafter, an optimization technique is used to search the optimal control parameter setting for the desired response.

Optimization of ECDM process using GA has been carried out in this research work. Mathematical models to correlate the responses and the control parameters are developed using RSM. The desired responses are minimum radial overcut (ROC) and minimum heat affected zone (HAZ) while the control parameters are applied voltage, electrolyte concentration and inter-electrode gap.

\section{LITERATURE REVIEW}

Mukherjee and Ray [3] reviewed application potential of several modeling and optimization techniques in metal cutting processes and presented a generic framework for parameter optimization in metal cutting processes for the benefits of selection of an appropriate approach. Experimental methods increase the cost of investigation. At times it is not feasible to perform all the experiments specially when the parameters and their levels are more in number. Wuthricha and Fasciob [4] developed mathematical model for correlating the interactions of some drilling control parameters and their effects on some responses using RSM. Kolahan and Bironro [5] used experimental results to develop the regression models for the different process characteristics in powder mixed electric discharge machining. Kuriakose and Shunmugam [6] used multiple regression model 
to represent relationship between input and output variables in a wire EDM process [6]. Karthikeyan et al. [7] conducted general factorial experiments to provide an exhaustive study of parameters of micro electric discharge milling process. Taguchi method is used for experiment design to optimize the cutting parameters. RSM is employed to design the experiments with a reduced number of experimental runs to achieve optimum responses. Lalwani et al. [8] applied RSM to investigate the effect of cutting parameters on surface roughness in finish hard turning operation.

Traditional optimization techniques fail to solve problems specially when the objective functions and constraints are complicated and implicit functions of the decision variables. Hence, a robust optimization technique which is immune with respect to production tolerances is desirable [9]. Yusup et al. [10] discussed evolutionary techniques and basic methodology of each technique in optimizing machining process parameters for both traditional and modern machining. As observed in the literature, application of evolutionary techniques in optimizing machining process parameters give good results. Samanta and Chakraborty [11] proved the applicability and suitability of evolutionary algorithm in enhancing the performance measures of nontraditional machining processes.

Unlike conventional optimization techniques, GA is a robust, global and is not bound to domainspecific heuristics. Jain et al. [12] used GA for optimization of process parameters of mechanical type advanced machining processes. Tansela et al. [13] proposed Genetically Optimized Neural Network System (GONNS) for selection of optimal cutting conditions for micro end milling operation. Singh and Rao [14] presented a multiobjective optimization technique based on GA to optimize the cutting parameters in turning processes. Zain et al [15] applied GA to optimize cutting conditions for minimizing surface roughness in end milling machining. Wang et al. [16] optimized multi-pass milling in terms of two objectives, i.e. machining time and production cost using an advanced search algorithm called parallel genetic simulated annealing (PGSA) to obtain the optimal cutting parameters. Singh and Rao [17] optimized the cutting parameters in turning processes using GA. Two conflicting objectives, i.e. tool life and operation time, have been simultaneously optimized.

The main objective of the present work is to determine the optimal machining parameters which minimize the objective functions of ROC and HAZ of the concerned ECDM process while machining silicon nitride ceramics. Process control parameters considered are applied voltage, electrolyte concentration and interelectrode gap.

\section{MATHEMATICAL MODELING}

The first order model fails to provide the interaction among different parameters while the third order models are difficult to solve. Hence, based on second order polynomial equation, Sarkar et al. [18] developed mathematical models for correlating ECDM process parameters while machining silicon nitride ceramics. The process control parameters considered are namely applied voltage, electrolyte concentration and inter-electrode gap while the desired responses are minimum radial overcut (ROC) and minimum heat affected zone (HAZ).

Table 1 lists the values for process control parameters with five levels for each parameter. A sum of twenty experimental runs is designed using Center composite design. The combinatorial effects of process control parameters at different levels on the measured responses are listed in Table 2.

Table 1 Coded and Actual control parameter values at different levels

\begin{tabular}{|l|c|c|c|c|c|}
\hline \multirow{2}{*}{} & \multicolumn{5}{|c|}{ Levels } \\
\cline { 2 - 6 } & 1 & 2 & 3 & 4 & 5 \\
\hline Coded value & -1.682 & -1 & 0 & 1 & 1.682 \\
\hline Applied Voltage (V) & 50 & 54 & 60 & 66 & 70 \\
\hline Electrolyte concentration (wt\%) & 10 & 14 & 20 & 26 & 30 \\
\hline Inter-electrode gap (mm) & 20 & 24 & 30 & 36 & 40 \\
\hline
\end{tabular}


Table 2 Design of experiments matrix showing coded values and observed responses

\begin{tabular}{|c|c|c|c|c|c|}
\hline \multirow[b]{2}{*}{$\begin{array}{l}\text { Sl. } \\
\text { No }\end{array}$} & \multicolumn{3}{|c|}{ Coded values of parameters } & \multicolumn{2}{|c|}{ Actual values of Responses } \\
\hline & $\begin{array}{c}\text { Applied } \\
\text { Voltage } \\
\text { (V) }\end{array}$ & $\begin{array}{c}\text { Electrolyte } \\
\text { concentration } \\
(\mathrm{wt} \%)\end{array}$ & $\begin{array}{l}\text { Inter-electrode gap } \\
\qquad(\mathrm{mm})\end{array}$ & $\begin{array}{l}\text { Radial overcut } \\
\text { (mm) }\end{array}$ & $\begin{array}{l}\text { Heat affected zone } \\
\qquad(\mathrm{mm})\end{array}$ \\
\hline 1 & -1 & -1 & -1 & 0.2045 & 0.0987 \\
\hline 2 & 1 & -1 & -1 & 0.2690 & 0.1192 \\
\hline 3 & -1 & 1 & -1 & 0.1416 & 0.0736 \\
\hline 4 & 1 & 1 & -1 & 0.2476 & 0.1030 \\
\hline 5 & -1 & -1 & 1 & 0.2020 & 0.0981 \\
\hline 6 & 1 & -1 & 1 & 0.1663 & 0.0889 \\
\hline 7 & -1 & 1 & 1 & 01362 & 0.0610 \\
\hline 8 & 1 & 1 & 1 & 0.2672 & 0.1153 \\
\hline 9 & -1.682 & 0 & 0 & 0.0996 & 0.0543 \\
\hline 10 & 1.682 & 0 & 0 & 0.3746 & 0.1264 \\
\hline 11 & 0 & -1.682 & 0 & 0.2432 & 0.1013 \\
\hline 12 & 0 & 1.682 & 0 & 0.1899 & 0.0983 \\
\hline 13 & 0 & 0 & -1.682 & 0.1866 & 0.0923 \\
\hline 14 & 0 & 0 & 1.682 & 0.1826 & 0.0623 \\
\hline 15 & 0 & 0 & 0 & 0.1836 & 0.0673 \\
\hline 16 & 0 & 0 & 0 & 0.2379 & 0.0764 \\
\hline 17 & 0 & 0 & 0 & 0.2566 & 0.0998 \\
\hline 18 & 0 & 0 & 0 & 0.1799 & 0.0805 \\
\hline 19 & 0 & 0 & 0 & 0.2165 & 0.0746 \\
\hline 20 & 0 & 0 & 0 & 0.1998 & 0.0723 \\
\hline
\end{tabular}

The mathematical model for correlating the radial overcut (ROC) and the process control parameters is developed as :

$$
\begin{aligned}
Y u(\mathrm{ROC}) & =0.16114+0.05333 x_{1}-0.01017 x_{2}-716 x_{3}+0.02454 x_{1}{ }^{2}+0.01727 x_{2}{ }^{2}+0.00598 x_{3}{ }^{2} \\
& +0.02603 x_{1} x_{2}-0.00940 x_{1} x_{3}+0.01493 x_{2} x 3 .
\end{aligned}
$$

Similarly the model for heat affected zone (HAZ) is developed as:

$$
\begin{aligned}
Y u(\mathrm{HAZ}) & =0.07835+0.01583 x_{1}-0.00418 x_{2}-0.00599 x_{3}+0.00523 x_{1}{ }^{2}+0.00857 x_{2}{ }^{2}+0.000618 x_{3}{ }^{2} \\
& +0.00905 x_{1} x_{2}-0.0006 x_{1} x_{3}+0.00382 x_{2} x_{3} .
\end{aligned}
$$

Where $x_{1}, x_{2}$ and $x_{3}$ are the process control parameters namely applied voltage, electrolyte concentration and inter-electrode gap respectively. $Y u(\mathrm{ROC})$ and $Y u(\mathrm{HAZ})$ are the responses for radial overcut and heat affected zone respectively. The effects of linear, higher order and the interaction of the independent process control parameters are represented in equations (1) and (2).

\section{SINGLE OBJECTIVE OPTIMIZATION}

\subsection{Optimization using GA}

GA is an evolutionary algorithm technique which borrows the idea of survival of the fittest amongst an interbreeding population to create a search strategy. It uses only the fitness value and no other knowledge is required for its operation. It is a robust search technique different to the problem solving methods used by more traditional algorithms which tend to be more deterministic in nature and get stuck up at local optima. The three basic operators of GA are reproduction, crossover and mutation. Initially a finite population of feasible solutions to a specified problem is maintained. Through reproduction, it then iteratively creates new populations from the old by ranking the solutions according to their fitness values. Crossover leads to interbreeding the fittest solutions to create new offsprings which are optimistically closer to the optimum solution to the problem at hand. As each generation of solutions is produced, the weaker ones fade away without producing offsprings, while the stronger mate, combining the attributes of both parents, to produce new and perhaps unique offsprings to continue the cycle. Occasionally, mutation is introduced into one of the solution strings to further diversify the population in search for a better solution. 
The present research work optimizes the desired response and control parameters by writing the mathematical models as developed in equations 1 and 2 as .M-files and then solved by GA using the MATLAB software. The initial population size considered while running the GA is 20 . A test of 10 runs with 50 generations each was conducted and the results are listed in Tables 3 and 4 for radial overcut and heat affected zone respectively. Table 3 lists the values of control parameters predicted using GA while searching for the optimal combination of the values in order to minimize the ROC. During the search, the response improved linearly with the number of initial population size. The best response was measured with population size 20 after which no improvement in the response value were recorded upon further increase of population size.

Table 3 GA predicted results for minimum ROC

\begin{tabular}{|c|c|c|c|c|}
\hline \multirow{2}{*}{ Trial number } & \multicolumn{3}{|c|}{ Control parameters } & \multirow{2}{*}{$\begin{array}{c}\text { Radial overcut } \\
\text { (mm) }\end{array}$} \\
\cline { 2 - 4 } & $\begin{array}{c}\text { Applied Voltage } \\
(\mathrm{V})\end{array}$ & $\begin{array}{c}\text { Electrolyte } \\
\text { concentration } \\
(\mathrm{wt} \%)\end{array}$ & $\begin{array}{c}\text { Inter-electrode gap } \\
(\mathrm{mm})\end{array}$ & \\
\hline 1 & 70 & 30 & 20 & 0.213694 \\
\hline 2 & 70 & 26 & 20 & 0.201163 \\
\hline 3 & 70 & 26 & 40 & 0.080134 \\
\hline 4 & 66 & 30 & 20 & 0.236961 \\
\hline 5 & 66 & 26 & 20 & 0.198345 \\
\hline 6 & 66 & 26 & 24 & 0.182563 \\
\hline 7 & 60 & 20 & 24 & 0.162851 \\
\hline 8 & 60 & 26 & 20 & 0.099862 \\
\hline 9 & 54 & 20 & 20 & 0.099862 \\
\hline 10 & 50 & 20 & 20 & 0.050088 \\
\hline
\end{tabular}

Fig. 1 shows the minimum ROC predicted using GA to be $0.050088 \mathrm{~mm}$. While running the GA, the results showed no improvement after 50 generations, hence the plot shows results only for 50 generations.

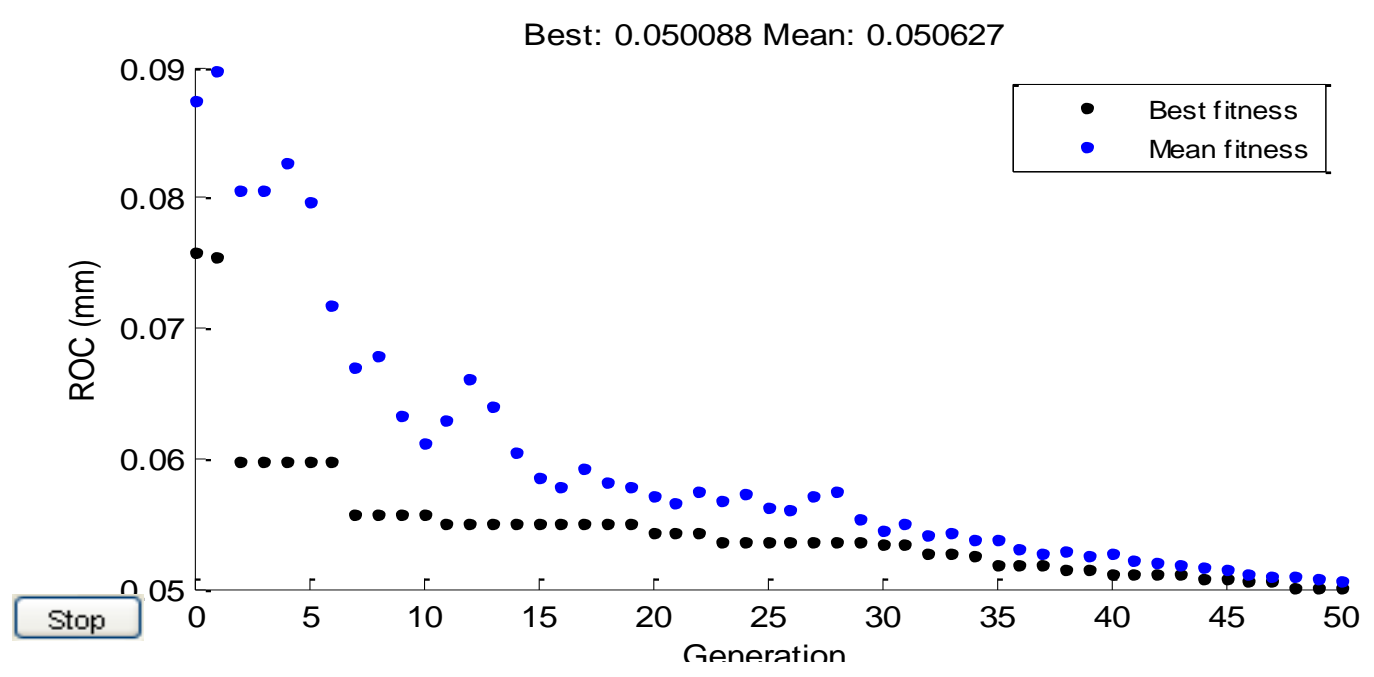

Fig. 1 Plot for GA predicted results for radial overcut

Similarly, Table 4 lists the values of control parameters while searching for the optimal combination of the values in order to minimize the HAZ. The best response was measured with population size 20 after which no improvement in the response value were recorded on further increase of population size. 
Table 4 GA predicted results for minimum HAZ

\begin{tabular}{|c|c|c|c|c|}
\hline \multirow{2}{*}{$\begin{array}{c}\text { Trial } \\
\text { number }\end{array}$} & $\begin{array}{c}|c| \\
\text { Applied Voltage } \\
(\mathrm{V})\end{array}$ & $\begin{array}{c}\text { Control parameters } \\
\text { Electrolyte } \\
\text { concentration } \\
(\mathrm{wt} \%)\end{array}$ & $\begin{array}{c}\text { Inter-electrode gap } \\
(\mathrm{mm})\end{array}$ & $\begin{array}{c}\text { Heat affected } \\
\text { zone } \\
(\mathrm{mm})\end{array}$ \\
\hline 1 & 70 & 30 & 20 & 0.143896 \\
\hline 2 & 70 & 10 & 24 & 0.121368 \\
\hline 3 & 66 & 10 & 30 & 0.098995 \\
\hline 4 & 66 & 14 & 30 & 0.091243 \\
\hline 5 & 60 & 14 & 36 & 0.085631 \\
\hline 6 & 60 & 20 & 36 & 0.054138 \\
\hline 7 & 54 & 26 & 36 & 0.063126 \\
\hline 8 & 54 & 26 & 40 & 0.064823 \\
\hline 9 & 50 & 20 & 40 & 0.059831 \\
\hline 10 & 50 & 20 & 40 & 0.042043 \\
\hline
\end{tabular}

Fig. 2 shows the minimum HAZ predicted using GA to be $0.042043 \mathrm{~mm}$. While running the GA, the results showed no improvement after 50 generations, hence the plot shows results only for 50 generations.

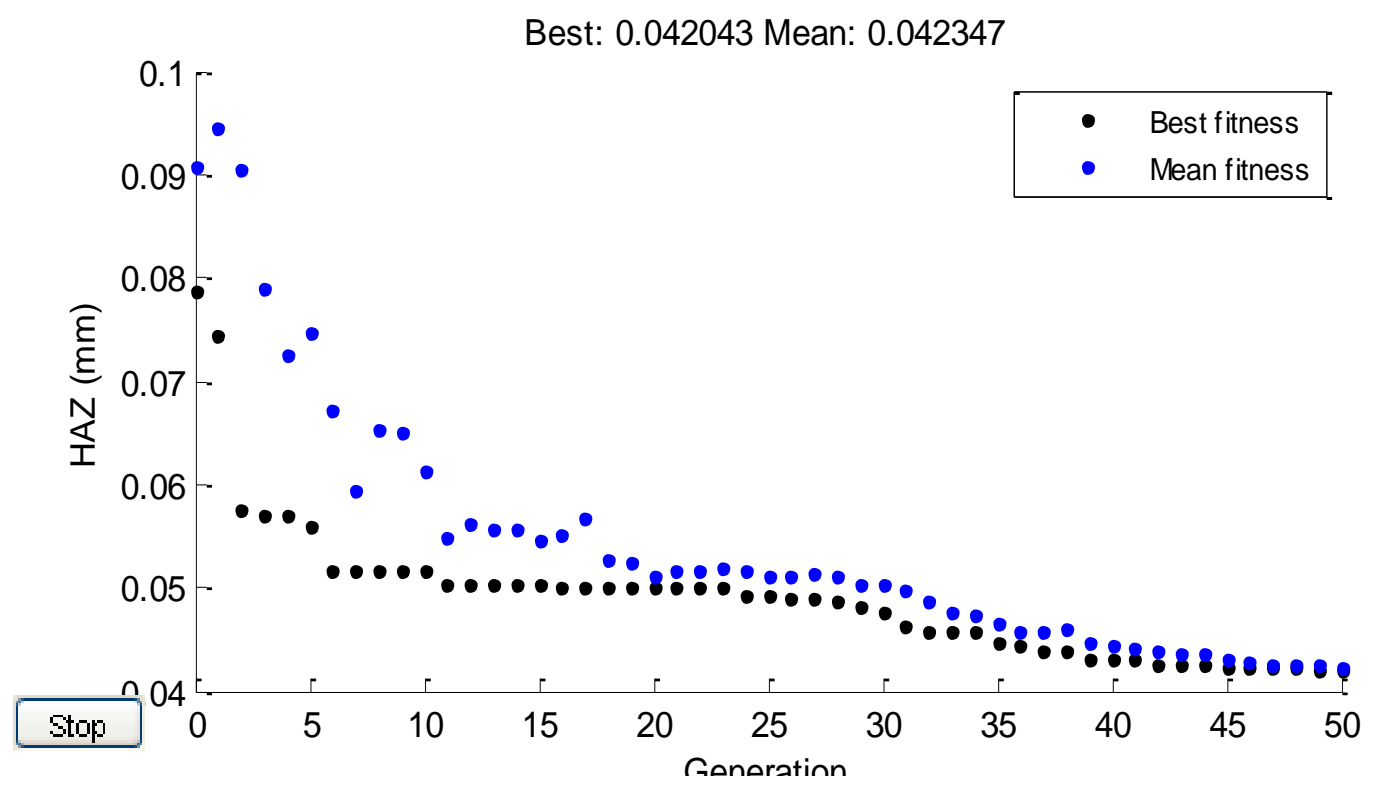

Fig. 2 Plot for GA predicted results for heat affected zone

\subsection{Validation of GA predicted results}

Validation of the GA predicted results is required to know the variation from the actual experimental results. The difference in results is measured by the factor percentage of prediction error.

The percentage of prediction error is calculated as

Prediction error\%

$$
=\frac{\text { Experiment al result }-\mathrm{GA} \text { predicted result }}{\text { Experiment al result }} \times 100
$$

In order to validate the test results predicted by GA, six random experimental results are compared with the GA predicted results as shown in Table 5. 
Table 5 Comparison of Experimental and GA predicted result

\begin{tabular}{|c|c|c|c|c|c|c|}
\hline $\begin{array}{c}\text { Sl. } \\
\text { No. }\end{array}$ & \multicolumn{2}{|c|}{ Experimental result } & \multicolumn{2}{c|}{$\begin{array}{c}\text { Gercentage prediction error } \\
(\%)\end{array}$} \\
\hline & $\begin{array}{l}\text { ROC } \\
(\mathrm{mm})\end{array}$ & $\begin{array}{c}\text { HAZ } \\
(\mathrm{mm})\end{array}$ & $\begin{array}{l}\text { ROC } \\
(\mathrm{mm})\end{array}$ & $\begin{array}{c}\text { HAZ } \\
(\mathrm{mm})\end{array}$ & $\begin{array}{c}\text { ROC } \\
(\mathrm{mm})\end{array}$ & $\begin{array}{c}\text { HAZ } \\
(\mathrm{mm})\end{array}$ \\
\hline 1 & 0.0996 & 0.0543 & 0.0968 & 0.0516 & 2.81 & 4.97 \\
\hline 2 & 0.1089 & 0.0623 & 0.1123 & 0.0611 & 3.12 & 1.92 \\
\hline 3 & 0.1362 & 0.0746 & 0.1299 & 0.0724 & 4.62 & 2.94 \\
\hline 4 & 0.1899 & 0.0805 & 0.1811 & 0.082 & 4.63 & 1.89 \\
\hline 5 & 0.2045 & 0.0889 & 0.2110 & 0.0862 & 3.17 & 3.03 \\
\hline 6 & 0.2690 & 0.0987 & 0.2598 & 0.0958 & 3.42 & 2.93 \\
\hline \multicolumn{6}{|c|}{ Average percentage prediction error } \\
\hline
\end{tabular}

On observation of results shown in Table 5, it is seen that both the individual and average prediction percentage error is well within acceptable limits thus proving the results predicted using GA to be valid.

The results listed in Table 5 is graphically plotted as shown in Fig. 3 and 4 to observe if there is any visual significant difference between the experimental and GA predicted results. The plots show similar progression with close proximity between the experimental and GA predicted results. Thus, GA proves to be a promising optimization technique for ECDM process.

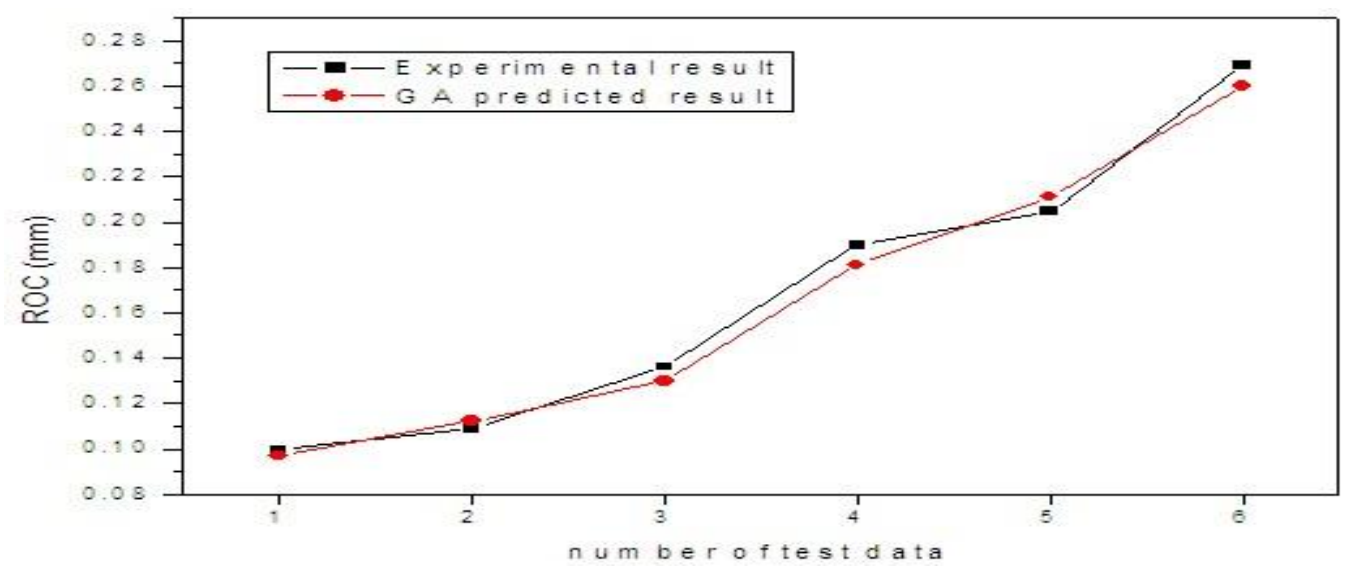

Fig. 3 Plot for comparison of experimental and GA predicted results for radial overcut

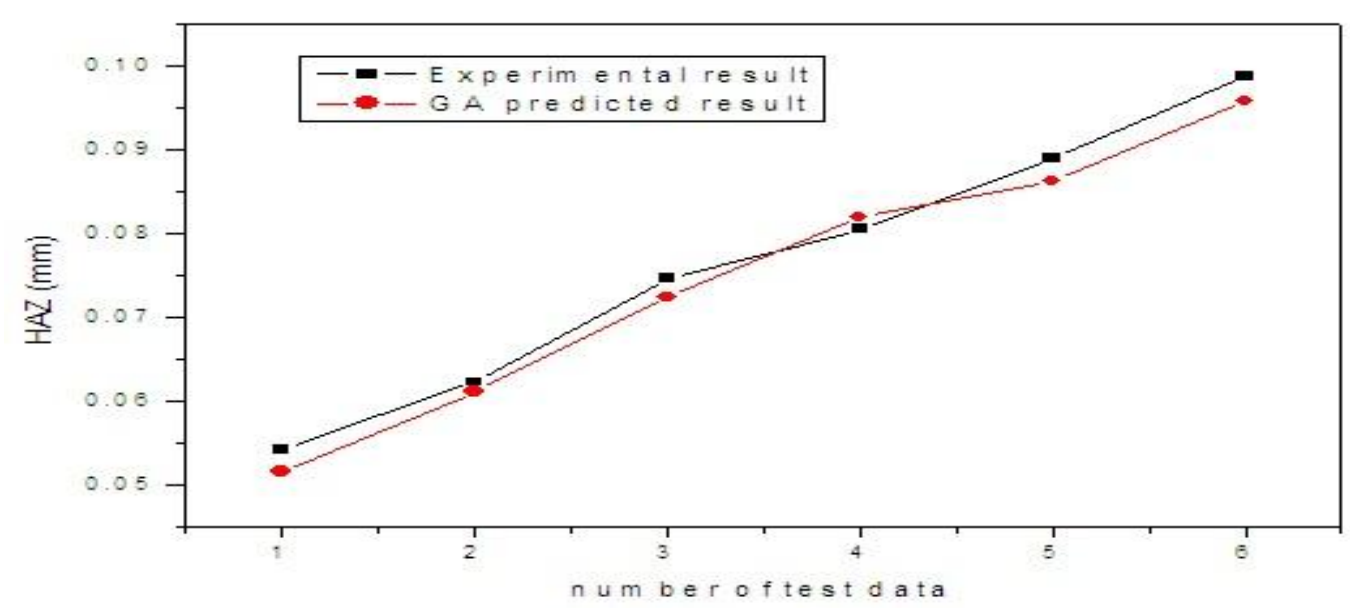

Fig. 4 Plot for comparison of experimental and GA predicted results for heat affected zone 


\section{MULTI-OBJECTIVE OPTIMIZATION}

It is difficult to search for a single solution that simultaneously optimizes conflicting objectives. While searching for solutions, an attempt to improve one objective leads to deterioration of the second objectives. An alternate solution during such cases is called non-dominated, Pareto optimal, if it cannot be eliminated by replacing it with another solution which improves an objective without worsening the other. The main objective when setting up and solving a multiobjective optimization problem is to find such non-dominated solutions.

Friedrich et al [19] performed runtime analyses and observed Multi Objective Evolutionary Algorithm have a marked preference for accepting quick small improvements. This leads to a faster search for new solutions close to the current population. Different types of multi objective GA developed for specific purpose differ from each other mainly by using specialized fitness functions and introducing methods to promote solution diversity. An elitist multiobjective GA ensures that the best solution does not deteriorate in the succeeding generations.

Eiben and Smit [20] obtained superior parameter values as well as information about problem instances, parameter values, and algorithm performance while using tuning algorithms. This information can serve as empirical evidence to justify design decisions. Lianga and Leung [21] used GA with adaptive elitistpopulation strategies for multimodal function optimization. Adaptive Elitist GA is shown to be very efficient and effective in finding multiple solutions. Zio and Bazzo [22] proposed a clustering procedure for reducing the number of representative solutions in the Pareto Front of multiobjective optimization problems. The result makes it easier for the decision maker to select the final solution on the basis of his or her assumed preferences. The clustering technique is shown to maintain the Pareto Front shape and relevant characteristics. Su and Hou [23] generated Pareto-optimal solutions using integrated multi population intelligent GA approach for the decision maker to determine the optimal parameters to assure a stable process and product qualities in the nanoparticle milling process.

The chief advantage of GA when applied to solve multi-objective optimization problems is the computation of an approximation of the entire Pareto front in a single algorithm run. Thus, considering these fore mentioned advantages of GA for solving multiobjective problems, it is applied to optimize the process of Electro chemical discharge machining while machining silicon nitride ceramics.

\subsection{Multiobjective Optimization using Genetic Algorithm}

GA is run in MATLAB for generating Pareto optimal solution points for minimizing radial overcut and heat affected zone while machining silicon nitride ceramics using ECDM. Equation for creating a fitness function for the multi objective optimization is written in a .M file. The range of the process parameters is placed as bounds on the three input control variables and the following algorithm options are set.

Selection function

Crossover function

Mutation function

Direction of migration

Distance measure function

Population size

: Tournament of size 2

: scattered

: Adaptive feasible

: Forward with migration function 0.2

: distance crowding

GA used to solve this multiobjective optimization problem is a controlled elitist genetic algorithm (a variant of NSGA-II). Elitist GA favors individuals with better fitness value. It maintains the diversity of population for convergence to an optimal Pareto front.

Weighted average change in the fitness function value over 100 generations is used as the criteria for stopping the algorithm. The optimized pareto front achieved is shown in Fig. 5. Input control parameters corresponding to each of the pareto optimal set of solutions are tabulated in Table 6 .

The two conflicting responses of minimizing radial overcut and heat affected zone are marked along $\mathrm{x}$ axis and y-axis respectively as shown in Figure 5. The star marks depict individual non dominated solution point among the pareto optimal set of all the star points which form the pareto front. The observed responses and the corresponding control parameter values are listed in Table 6. 


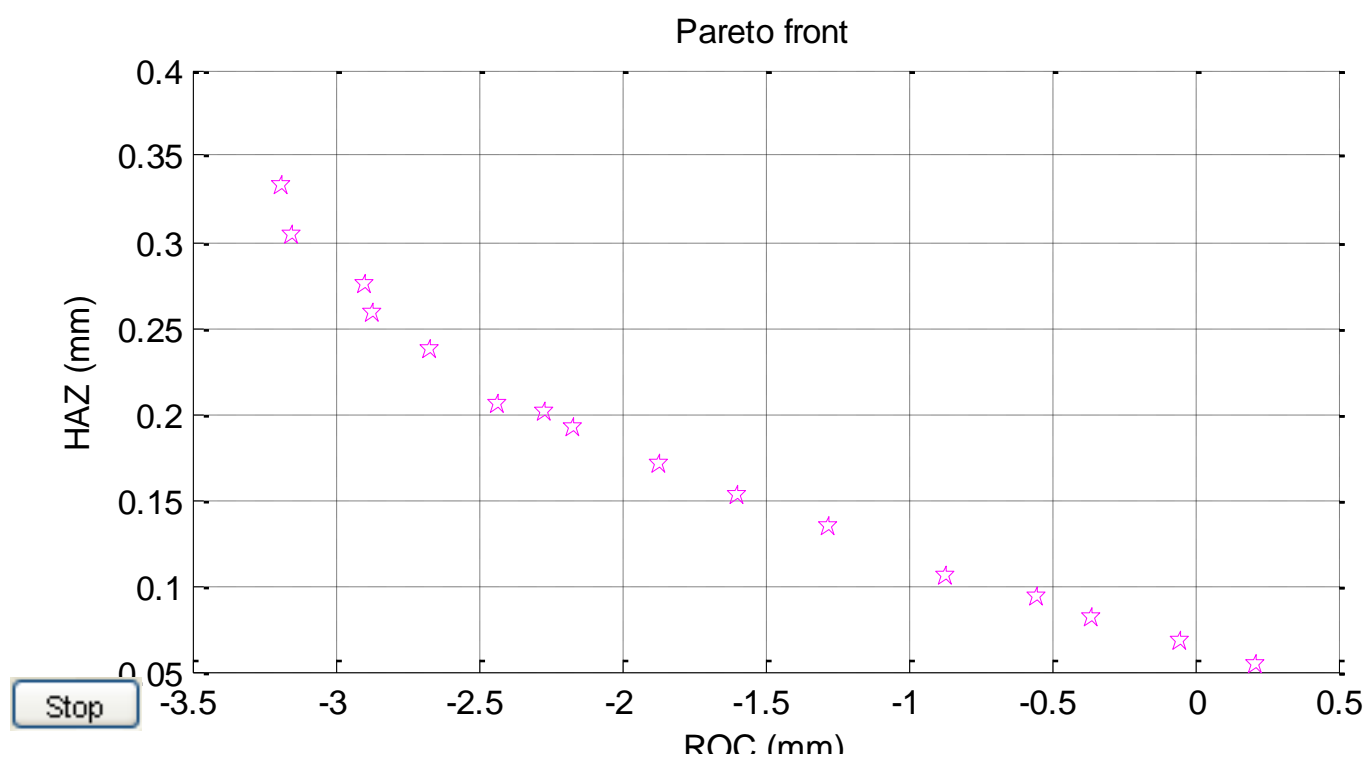

Fig. 5 GA predicted Pareto optimal solution

Table 6 Control parameter and Response values for Pareto optimal solution

\begin{tabular}{|c|c|c|c|c|c|}
\hline \multirow{3}{*}{ S1. No. } & \multicolumn{3}{|c|}{ Process control parameters } & \multicolumn{2}{c|}{ Responses } \\
\cline { 2 - 6 } & $\begin{array}{c}\text { Applied voltage } \\
(\mathrm{V})\end{array}$ & $\begin{array}{c}\text { Electrolyte concentration } \\
(\text { Wt. } \%)\end{array}$ & $\begin{array}{c}\text { Inter-electrode gap } \\
(\mathrm{mm})\end{array}$ & $\begin{array}{c}\text { ROC } \\
(\mathrm{mm})\end{array}$ & $\begin{array}{c}\text { HAZ } \\
(\mathrm{mm})\end{array}$ \\
\hline 1 & 70 & 30 & 20 & -3.45 & 0.34 \\
\hline 2 & 70 & 30 & 20 & -3.41 & 0.31 \\
\hline 3 & 70 & 30 & 20 & -2.91 & 0.28 \\
\hline 4 & 66 & 30 & 20 & -2.87 & 0.26 \\
\hline 5 & 66 & 30 & 20 & -2.69 & 0.24 \\
\hline 6 & 66 & 30 & 20 & -2.49 & 0.21 \\
\hline 7 & 60 & 26 & 24 & -2.32 & 0.2 \\
\hline 8 & 60 & 26 & 24 & -2.28 & 0.159 \\
\hline 9 & 60 & 26 & 24 & -1.87 & 0.17 \\
\hline 10 & 54 & 26 & 24 & -1.62 & 0.15 \\
\hline 11 & 54 & 26 & 24 & -1.38 & 0.14 \\
\hline 12 & 54 & 26 & 30 & -0.88 & 0.11 \\
\hline 13 & 50 & 20 & 30 & -0.61 & 0.059 \\
\hline 14 & 50 & 20 & 30 & -0.42 & 0.058 \\
\hline 15 & 50 & 20 & 30 & 0.28 & 0.052 \\
\hline 16 & 50 & 20 & & & 0.051 \\
\hline
\end{tabular}

It is observed that an improvement in minimizing radial overcut deteriorates the quality of heat affected zone and vice versa. Thus, each solution point is a unique non dominated solution point.

\section{RESULT AND ANALYSIS}

While machining silicon nitride ceramics using ECDM process, two objectives, radial overcut and heat affected zone are considered to be important as they affect the quality of the product. While optimizing the responses individually, the GA predicted value of minimum radial overcut is $0.050088 \mathrm{~mm}$ as shown in Fig. 1.The corresponding control parameter values are $50 \mathrm{~V}$ applied voltage, electrolyte concentration of $20 \mathrm{wt} \%$ and an inter-electrode gap of $20 \mathrm{~mm}$. Compared to the experimental result, GA shows an improvement of 0.049512 $\mathrm{mm}$ while minimizing radial overcut.

On observation of GA predicted combination of control parameter values shown in Table 3, it is seen that applied voltage is directly proportional to ROC. Higher applied voltage results in larger ROC. Medium electrolyte concentration provides a low ROC. The ROC decreases as the inter-electrode gap increases. If high voltage is to be used, then the inter-electrode gap should also be high. Overall, in order to achieve minimum 
radial overcut the control parameters are to be set at low applied voltage and medium electrolyte concentration and a medium inter-electrode gap.

Similarly, the minimum HAZ predicted using GA is $0.0543 \mathrm{~mm}$ as shown in Fig. 2, with the corresponding control parameter values of $50 \mathrm{~V}$ applied voltage, electrolyte concentration of $20 \mathrm{wt} \%$ and an inter-electrode gap of $30 \mathrm{~mm}$. Again, on comparison GA shows an improvement of $0.012257 \mathrm{~mm}$ against the experimental result. The GA predicted results as listed in Table 4 shows that HAZ increases as the applied voltage increase. HAZ is inversely proportional to the inter-electrode gap. As the gap increases, the HAZ decrease. Optimal control parameters for minimum HAZ is obtained by using low applied voltage and medium values of electrolyte concentration and inter-electrode gap.

The average percentage prediction error of GA when compared with the experimental results as shown in Table 5 is $3.62 \%$ and $2.94 \%$ for radial overcut and heat affected zone respectively. Thus, the GA predicted results are within acceptable limits (confidence level > 95\%) proving the validity of the GA as an appropriate optimization technique for ECDM process.

In real life situations, as in this case of multiobjective optimization of ECDM process, the responses often conflict with each other. At such situations it is often difficult and at times impossible to predict a single solution point that optimizes both the responses. Pareto optimal set of solution provides a novel approach for solving such problems. This multi objective problem is optimized using the multiobjective GA in MATLAB software. The solution obtained is a set of pareto optimal points as shown in Fig. 5 where each point is non dominated. The observed responses were obtained in a single process parametric combination setting. Table 6 records the range of values for responses at different parametric combination. From the response values as listed in Table 6, it is observed that an improvement in minimizing ROC deteriorates the quality of HAZ and vice versa. Thus, each solution point is a unique non dominated solution point. Instead of a single solution point, a set of solution points are predicted for simultaneously optimizing both the responses. A change in the value of any one of the considered control parameters further improves any one of the responses at the cost of degrading the second response. This result is helpful as it provides a wide range of optimal setting of control parameters for simultaneously optimizing both the responses. Hence, flexibility in the operation of the machine is achieved by presenting different parametric combinations for the range of predetermined desired responses.

\section{CONCLUSION}

ECDM process provides an excellent method for machining Silicon nitride ceramics. The desired responses required are minimum ROC and minimum HAZ. Both the responses are a measure of machining efficiency and quality of machining process. Thus, it is a min-min two objective optimization problem. Solution to such optimization problems is best described by a set of pareto optimal non dominated points as presented in this research work. The decision maker is provided with the choice of trade off between these two objectives which further increase the flexibility to select the optimal cutting parameters.

\section{REFERENCES}

[1] Mediliyegedara,T.K.K.R., De Silva,A.K.M., Harrison,D.K. and McGeough,J.A. "An intelligent pulse classification system for electro-chemical discharge machining (ECDM) - a preliminary study," Journal of Materials Processing Technology, 2004, vol.149, pp.499-503.

[2] Wuthricha,R. and Fasciob, V. "Machining of non-conducting materials using electrochemical discharge phenomenon - an overview," International Journal of Machine Tools \& Manufacture,2005, vol.45, pp. 1095-1108.

[3] Mukherjee, I., Ray,P. Kumar. "A review of optimization techniques in metal cutting processes," Computers \& Industrial Engineering, 2006, vol. 50, pp. 15-34.

[4] Onwubolu, G.C. and Kumar, S."Response Surface Methodology based approach to CNC drilling operations," Journal of Materials Processing Technology, 2006, vol.171, pp. 41- 47.

[5] Farhad Kolahan and Mohammad Bironro "Modeling and Optimization of Process Parameters in PMEDM by Genetic Algorithm," World Academy of Science, Engineering and Technology, 2008, vol. 48, pp. 2070-3740.

[6] Shajan Kuriakose and Shunmugam, M.S. "Multi-objective optimization of wire-electro discharge machining process by Non-Dominated Sorting Genetic Algorithm," Journal of Materials Processing Technology, 2005, vol.170, pp.133-141.

[7] Karthikeyan, G., Ramkumar J., Dhamodaran S., Aravindan S. "Micro electric Discharge Milling process performance: An experimental investigation," International Journal of Machine Tools \& Manufacture, 2010 , vol. 50, pp. 718-727.

[8] Lalwani, D.I., Mehta N.K., Jain P.K."Experimental investigations of cutting parameters influence on cutting forces and surface roughness in finish hard turning of MDN250 steel,"Journal of materials processing technology, 2008, vol. 206, pp.167-179.

[9] Yildiz, Ali R. "A comparative study of population-based optimization algorithms for Turning Operations," Information Sciences, 2012, vol. 210, pp. 81-88.

[10] Norfadzlan Yusup, Azlan Mohd Zain, Siti Zaiton Mohd Hashim,"Evolutionary Techniques in optimizing machining parameters: Review and recent applications (2007- 2011)," Expert Systems with Applications, 2012, vol. 39, pp. 909-9927. 
[11] Suman Samanta, Shankar Chakraborty, "Parametric optimization of some non- Traditional machining processes using artificial bee colony algorithm," Engineering Applications of Artificial Intelligence, 2011, vol. 24, 2011, pp. 946-957.

[12] Neelesh K. Jain, V.K. Jain, Kalyanmoy Deb. " Optimization of process parameters of mechanical type advanced machining processes using genetic algorithms," International Journal of Machine Tools \& Manufacture, 2007, vol. 47, pp. 900-919.

[13] Tansela, I.N., Ozcelik, B., Bao, W.Y., Chen, P., Rincon, D., Yang, S.Y. and Yenilmez, A. "Selection of optimal cutting conditions by using GONNS", International Journal of Machine Tools \& Manufacture, 2006, Vol. 46, pp. 26-35.

[14] Singh, D. and Rao, P.V. "Optimization of tool geometry and cutting parameters for hard turning", Materials and Manufacturing Processes, 2007, Vol. 22, pp.15-21.

[15] Zain, A.M., Haron, H., and Sharif, S. "Application of GA to optimize cutting conditions for minimizing surface roughness in end milling machining process", Expert Systems with Applications, 2010, Vol 37, pp. 4650-4659.

[16] Wang, Z.G., Wong,Y.S., Rahman, M. and Sun,J. "Multi-objective optimization of high- speed end milling with parallel genetic simulated annealing," International Journal of Machine tools and Manufacture, 2006, vol. 31, pp.209-218.

[17] Singh, D. and Rao, P.V. "Optimization of tool geometry and cutting parameters for hard turning", Materials and Manufacturing Processes,2007, vol. 22, pp.15-21.

[18] Sarkar, B.R.,Doloi,B.and Bhattacharyya, B. Parametric analysis on electrochemical discharge machining of Silicon Nitride ceramics, International Journal of Advanced Manufacturing Technology, 2006, vol.28, pp.873-881.

[19] Friedrich, T., Horobab, C. and Neumanna, F. "Illustration of fairness in evolutionary multi-objective optimization", Theoretical Computer Science, 2011, vol. 412, pp. 15461556.

[20] Eiben, A.E. and Smit S.K. " Parameter tuning for configuring and analyzing evolutionary algorithms", Swarm and Evolutionary Computation, 2011, Vol. 1, pp.19-31.

[21] Lianga, Y. and Leung, K.-S."Genetic Algorithm with adaptive elitist-population strategies for multimodal function optimization”, Applied Soft Computing, 2011, Vol.11, pp. 2017-2034.

[22] Zio, E. and Bazzo, R. (2011) “ A clustering procedure for reducing the number of representative solutions in the Pareto Front of multiobjective optimization problems", vol.210, 2011, pp. 624-634.

[23] Su,C-H. and Hou T-H. "Using multi-population intelligent genetic algorithm to find the pareto-optimal parameters for a nano-particle milling process", Expert Systems with applications, 2008, Vol. 34, pp. 2502-2510. 